\title{
Perfil profissional do egresso do Programa de Pós-Graduação em Odontopediatria da Faculdade de Odontologia da USP-São Paulo
}

\author{
Julianna de Oliveira Lima Parizotto*; José Carlos Petorossi Imparato**; Tatine Fernandes \\ Novaes $^{* * *}$ \\ * Graduada pela Faculdade de Odontologia da Universidade de \\ São Paulo (FOUSP) \\ ** $\quad$ Professor Livre Docente da Disciplina de Odontopediatria da \\ Faculdade de Odontologia da Universidade de São Paulo \\ (FOUSP) e Professor do Programa de Pós-Graduação da Facul- \\ dade São Leopoldo Mandic \\ *** Mestre e Doutora em Odontopediatria pela Faculdade de Odon- \\ tologia da Universidade de São Paulo (FOUSP) e Pós Douto- \\ randa em Odontopediatria na Universidade Cruzeiro do Sul \\ (UNICSUL)
}

\section{RESUMO}

O objetivo deste trabalho foi avaliar o atual local e tipo de atuação profissional do egresso do programa de pós-graduação em Ciências Odontológicas com área de concentração em Odontopediatria da Faculdade de Odontologia da Universidade de São Paulo (campus São Paulo) - FOUSP. Para tanto, a relação dos egressos dos cursos de mestrado e doutorado na área de concentração em Odontopediatria da FOUSP foi solicitada junto à coordenadoria do programa de pós-graduação da instituição. A partir de então, o local e tipo de atuação profissional exercidos por cada um dos egressos foram avaliados por meio da Plataforma Lattes (Curriculum Lattes - CL) desde a conclusão do curso de pós-graduação por cada um deles, considerando as atualizações feitas até o mês de setembro de 2012. Para avaliação dos resultados foram considerados o gênero do participante, data de matrícula no curso de pós-graduação, data de defesa da dissertação e/ou tese, data de atualização do $C L$, local e tipo de atividade profissional exercida atualmente (docência ou clínica). Partindo de um total de 65 egressos (54 do gênero feminino e 11 do masculino), 44 exercem atividade relacionada à docência, sendo que a maioria deles atua em São Paulo e atualiza o $C L$ frequentemente. As atividades clínicas foram pouco relatadas no $C L$.

Descritores: Odontopediatria. Área de Atuação Profissional. Educação de PósGraduação em Odontologia.

\section{INTRODUÇÃO}

Os programas de pós-graduação dão continuidade aos cursos de graduação em odontologia, no qual os ex-acadêmicos de todo o país procuram as universidades brasileiras para a realização de cursos lato e stricto sensu ${ }^{1}$.

Os cursos de pós-graduação lato sensu são considerados cursos de especialização, ao passo que os stricto sensu são voltados à formação científica e acadêmica direcionados à pesquisa. $\mathrm{O}$ mestrado e o doutorado fazem parte da formação stricto sensu e implicam na elaboração de um projeto de pesquisa finalizado na apresentação de um trabalho de conclusão de curso (dissertação de mestrado ou tese de doutorado) ${ }^{1}$.

De acordo com a avaliação trienal 
da Coordenação de Aperfeiçoamento de Pessoal de Nível Superior (CAPES) que é uma instituição que oferece incentivo e fiscaliza os cursos de pós-graduação no país, até o ano de 2007, os programas de mestrado e doutorado na área de Odontologia no país, eram em número de 84. Desses, 12 representavam cursos de mestrado profissional, 25 de mestrado acadêmico, 46 de mestrado e doutorado e um programa oferecia apenas o doutorado. De acordo com a CAPES, o mestrado profissional é a designação do mestrado que enfatiza estudos e técnicas diretamente voltadas ao desempenho de um alto nível de qualificação profissional. Tal enfoque é a única diferença do mestrado profissional em relação ao acadêmico².

Esse panorama vem sofrendo mudanças. Segundo números mais recentes oferecidos pela CAPES, em 2009 houve um aumento significativo na formação de doutores em todo o país com a existência de 52 programas com nível de doutorado e apenas 18 cursos de mestrados profissionais ${ }^{2}$. Por outro lado, o mercado de trabalho nacional parece não acompanhar esse aumento na qualificação dos recursos humanos formados em odontologia. Assim, a falta de vagas pode tornar a atividade docente competitiva e mal remunerada, restando a muitos mestres e doutores dedicar-se à atividade clínica.

A partir das novas diretrizes curriculares propostas pela Lei de Diretrizes e Bases da Educação (LDB), foi definido para área odontológica que o curso de graduação em Odontologia deveria ter como perfil do egresso, uma formação generalista, humanista, crítica e reflexiva, para atuar em todos os níveis de atenção à saúde. Entretanto, a formação cada vez mais fragmentada contribui para formação de profissionais cada vez mais despreparados para o mercado de trabalho, influindo também nos cursos de pósgraduação ${ }^{3,4}$.

Com relação à distribuição geográfica dos cursos de pós-graduação, a maioria (75\%) se concentra na região Sudeste, em maior frequência no estado de São Paulo. Além disso, São Paulo é também o estado que forma $48 \%$ dos doutores brasileiros e produz $50 \%$ dos artigos científicos publicados em revistas indexadas ${ }^{5}$. A Universidade de São Paulo é responsável pela formação continuada de inúmeros profissionais que procuram pelos seus cursos de pós-graduação em diferentes áreas.

Assim, o objetivo deste trabalho foi avaliar o atual local e tipo de atuação profissional do egresso do programa de pósgraduação em Ciências Odontológicas com área de concentração em Odontopediatria da Faculdade de Odontologia da Universidade de São Paulo.

\section{MATERIAL E MÉTODOS}

Após a aprovação deste estudo pelo Comitê de Ética em Pesquisa local (Protocolo 34/11), a relação dos egressos dos cursos de mestrado e doutorado do programa de pós-graduação em Ciências Odontológicas com área de concentração em Odontopediatria da FOUSP foi solicitada junto à coordenadoria do programa de pós-graduação da instituição.

Um total de 65 egressos foram relacionados entre as matrículas efetivadas em cinco turmas (processos seletivos de 2000, 2002, 2004, 2006 e 2008). A partir de então, o local e tipo de atuação profissional exercida por cada um desses foram avaliados por meio da Plataforma Lattes (Curriculum Lattes $C L)$. O $C L$ de cada egresso foi acessado na íntegra (além do texto inicial fornecido pelo $C L$ ou individualizado pelo au- 
tor/pesquisador) para levantamento dos dados que constavam nos campos endereço e atuação profissional (relatos de vínculo de servidor público, celetista ou professor visitante). Foram considerados o período de conclusão do curso de pós-graduação e a data de atualização dos dados disponibilizados no CL de 2000 até o mês de setembro de 2012, considerando atualizações recentes ou currículos não atualizados.

Para análise dos resultados foram considerados o gênero do participante, data de matrícula no curso de pós-graduação, data de defesa da dissertação e/ou tese, data de atualização do $C L$, local e tipo de atividade profissional exercida atualmente (docência ou clínica).

\section{RESULTADOS}

A maioria dos egressos do programa de pós-graduação em Ciências Odontológicas com área de concentração em Odontopediatria da FOUSP é do gênero feminino estando, atualmente, vinculados à docência (Gráfico 1). No período avaliado foram defendidas 23 dissertações de mestrado e 42 teses de doutorado. Atividades clínicas não foram relatadas com clareza no CL. O local de atuação relatado com maior frequência foi o estado de São Paulo (Gráfico 2). A maioria dos currículos (78\%) foi atualizada recentemente (Gráfico 3).

Gráfico 1 - Avaliação da atividade profissional exercida na atualidade pelos egressos em geral (total de egressos $=65$ )

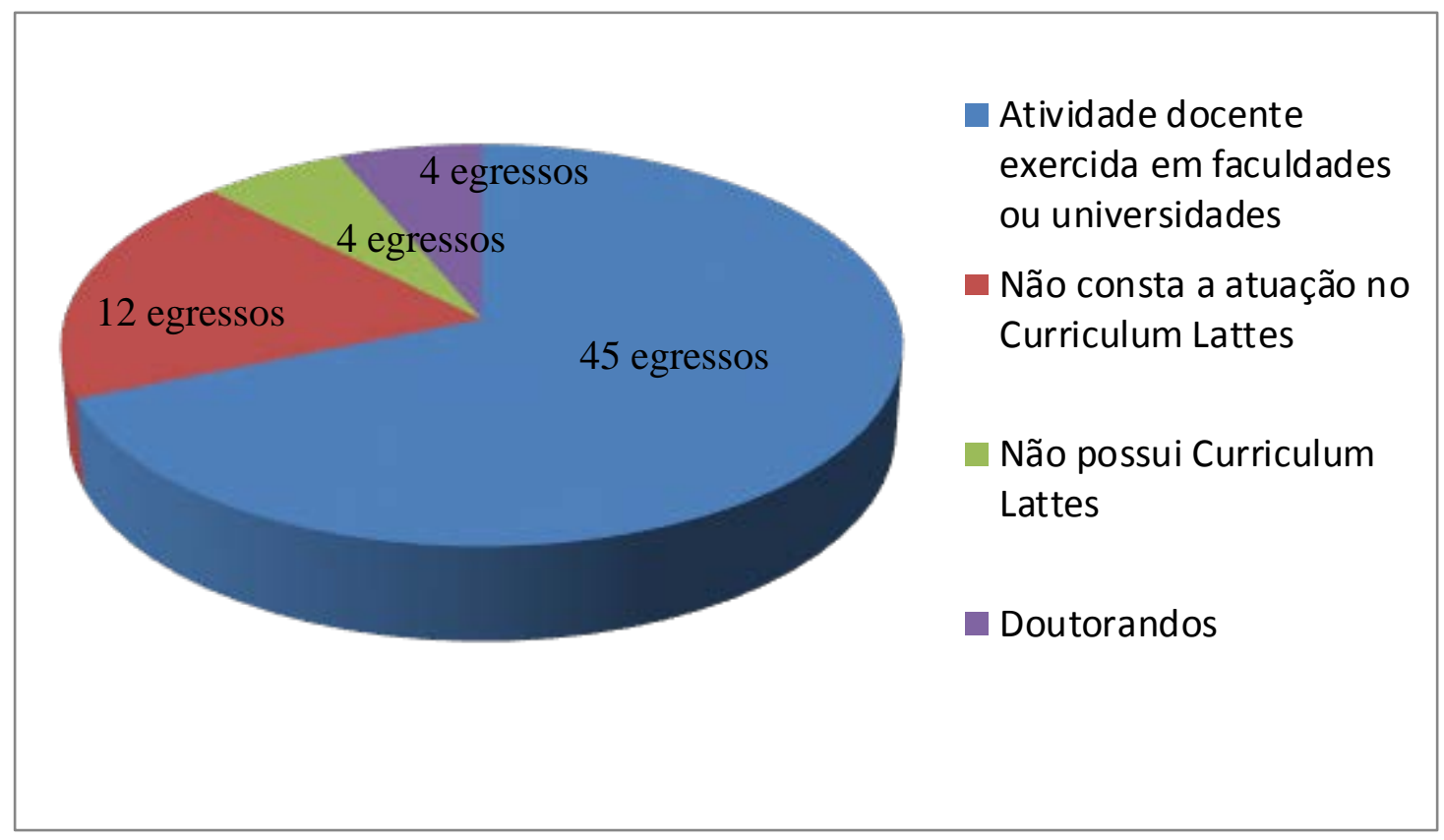


Perfil profissional do egresso do Programa de Pós-Graduação em Odontopediatria da Faculdade de Odontologia da USP-São Paulo

Gráfico 2 - Avaliação do local de atuação profissional do egresso que está ligado a uma universidade

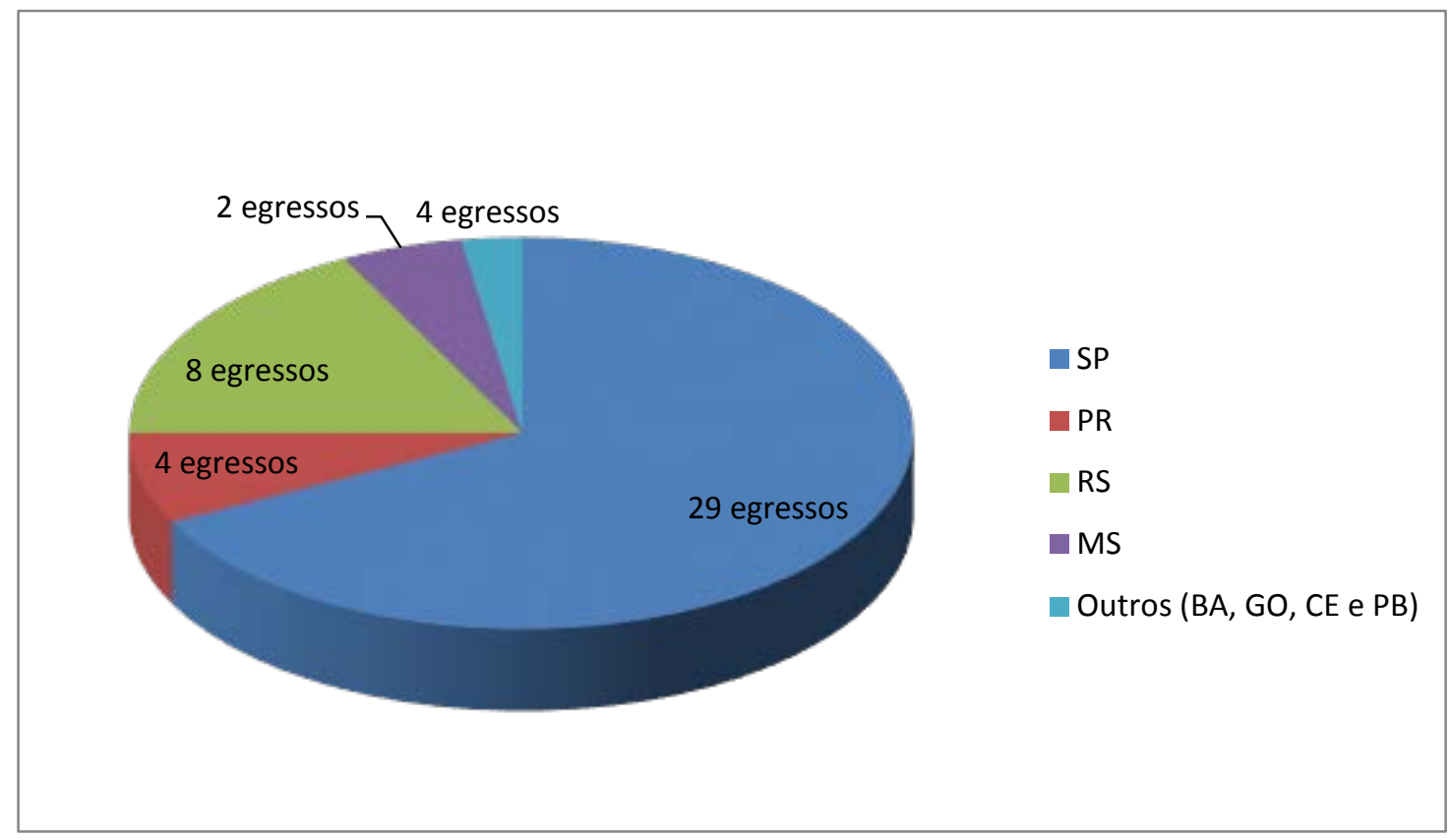

Gráfico 3 - Avaliação do período de atualização do CL (buscas feitas em setembro de 2012, totalizando 47 egressos que possuem Curriculum Lattes)

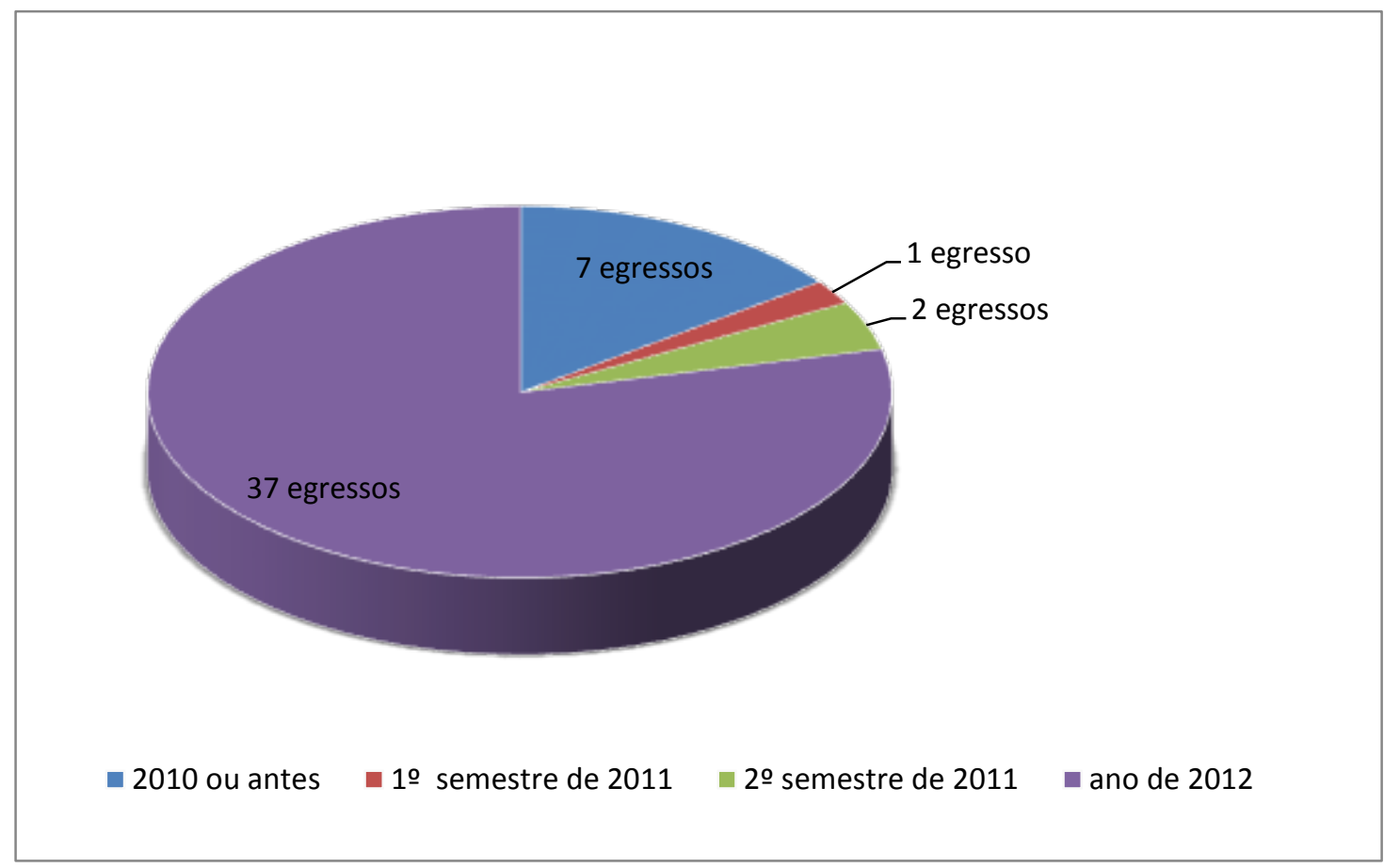

\section{DISCUSSÃO}

A Universidade de São Paulo é responsável pela formação de um grande número de mestres e doutores em odontologia (es- pecialidades diversas) oriundos de diversas regiões brasileiras. Assim, o presente trabalho teve como objetivo principal, avaliar o perfil profissional do egresso do programa de 
pós-graduação em Ciências Odontológicas com área de concentração em Odontopediatria da Faculdade de Odontologia da Universidade de São Paulo.

A partir dos gráficos apresentados neste estudo foi possível observar que a quantidade de egressos do programa de doutorado é consideravelmente maior do que a do programa de mestrado, sendo que das cinco turmas definidas para análise dos resultados, apenas uma turma (no ano de 2000) obteve maior número de egressos no programa de mestrado. Essa formação de mão de obra altamente qualificada é também encontrada em outros países. Castro ${ }^{6}$ relata um aumento de $15 \%$ por ano no número de doutores da América Latina. Considera-se também que naturalmente após a finalização do mestrado o aluno inicia o doutorado, apenas alguns não se encaixam nessa condição.

Acreditamos também que as exigências do Ministério da Educação (MEC) e instituições de ensino superior induzem os docentes à busca continuada por aperfeiçoamento profissional, o que possivelmente justifica uma maior procura e formação de doutores; muitos desses tendo também cursado previamente o mestrado.

Calculado como porcentagem do Produto Interno Bruto (PIB), o investimento do estado de São Paulo em Pesquisa e Desenvolvimento supera o de gigantes emergentes como a China, a Índia e o próprio Brasil ${ }^{5}$. Na existência desse incentivo financeiro, justifica-se o achado de um maior número de vagas em cursos de pós-graduação no estado de São Paulo, e consequentemente, o achado de um maior número de egressos do programa de ciências odontológicas da FOUSP vinculados à atividade docente em faculdades ou universidades situadas também no estado de São Paulo ${ }^{7}$.
Um número maior de cursos de pósgraduação stricto sensu em todo o Brasil, direciona outra preocupação voltada à qualidade da produção científica decorrente desses cursos de mestrado e doutorado. A aprovação de um maior número de vagas nesses cursos exige fiscalização e incentivo às Instituições sede, além de requerer apoio financeiro para custeio de estudos de maior rigor e qualidade metodológicos ${ }^{6}$.

O aumento no número de egressos de cursos stricto sensu requer também uma equivalência na oferta de vagas no mercado de trabalho, o que nem sempre acontece. Castro levanta a hipótese de, no futuro, existir uma grande quantidade de doutores em odontologia não vinculados ao ensino e/ou pesquisa $^{6}$. No presente trabalho, a amostra estudada mostra um perfil profissional que não corrobora essa informação, sendo a realização de atividades ligadas à universidades ou faculdades relatadas pela maioria dos egressos do programa de pós-graduação com área de concentração em Odontopediatria da Universidade de São Paulo.

Os presentes resultados mostram um número de egressos do gênero feminino muito maior do que do gênero masculino. De acordo com Nunes et al., profissionais do gênero feminino, na odontologia, são maioria no Brasil desde a década de 80 . Atualmente, as mulheres correspondem a $56 \%$ do total de Cirurgiões-Dentistas brasileiros ${ }^{7}$.

Após a análise dos Curriculum Lattes dos egressos do programa de pós-graduação na área de odontopediatria da FOUSP, verificou-se que grande parte deles não especifica detalhes da sua atuação, o que dificultou especialmente a análise da quantidade de egressos que atuam também na área clínica. Outra justificativa para tal dificuldade pode estar relacionada ao fato dos pós-graduandos 
serem bolsistas durante a pós-graduação e por isso, dedicando-se exclusivamente ao cumprimento das atividades do curso conforme normas estabelecidas na aceitação e outorga das bolsas de estudos, mantém-se afastados das atividades clínicas que, consequentemente, deixam de ser relatadas no Curriculum Lattes.

Atualmente o desenvolvimento de projetos, estudos, artigos e demais atividades profissionais podem ser resumidos na publicação do Curriculum Lattes. Assim, observamos uma grande preocupação com a atualização desse instrumento feita pela maioria dos egressos dos cursos de mestrado e doutorado pertencentes à nossa amostra, especialmente àqueles que estão vinculados à área acadêmica. Tal fato se relaciona à necessidade de apresentarem constante produção científica durante sua contratação e progressão de carreira ou até mesmo participação em concursos públicos já que o Curriculum Lattes é uma plataforma conhecida nacionalmente para sumarizar publicações e atividades exercidas.

\section{CONCLUSÃO}

A maioria dos egressos do curso de pós-graduação da FOUSP com área de concentração em odontopediatria exerce atividade docente no estado de São Paulo, sumarizando sua produção científica no CL de forma frequente.

\section{REFERÊNCIAS}

1. Morita MC, Haddad AE, Araújo ME. Perfil atual e tendências do CirurgiãoDentista brasileiro. Maringá: Dental Press; 2010.

2. Coordenação de Aperfeiçoamento de Pessoal de Nível Superior (CAPES). Brasília, DF. Disponível em: www.capes.gov.br
3. Vargas AMD, Vasconcelos MA. Construção da Clínica Integrada de atenção primária da Faculdade de Odontologia da Universidade Federal de Minas Gerais: A experiência da Clínica Integrada I. Arq. Odontol. 1998; 34:71-81.

4. Ministério da Educação e Cultura (MEC). Conselho Nacional de Educação. Resolução CNE/CES 3/2002. Diário Oficial da União, Brasília; 2002.

5. Marques F. A contribuição de São Paulo. Pesq. FAPESP, maio 2010; 171:28:35.

6. Castro F. O custo da ciência latinoamericana. Agência FAPESP, março 2007. Disponível em: http://agencia.fapesp.br/o_custo_da_cienci a_ latinoamericana/6858/

7. Nunes MF, Leles CR, Gonçalves MM. Gênero e escolha por especialidades odontológicas: estudo com egressos de uma universidade pública. Ver. Odontol. Bras. Central. 2010; 19(49):142-5.

\section{ABSTRACT \\ Professional profile of egress of the gradu- ate program in Pediatric Dentistry of School of Dentistry of University of São Paulo}

The aim of this study was to evaluate the professional profile (location and professional activity) of egress of the graduate program in Pediatric Dentistry of School of Dentistry of University of São Paulo. For this, the number and the contact of the egresses of Master's and $\mathrm{PhD}$ courses with concentration area in Pediatric Dentistry were requested with the coordinating body of the graduate program of the Institution. The location and the professional activity exercised by each of the egresses was evaluated by the Lattes Platform (Curriculum Lattes - CL) since the con- 
clusion of the graduate course, considering the updates of this system until September 2012.To describe the results, were considered the participant's gender, date of registry in the graduate program, date of the defense of dissertation and / or thesis, update date of the CL, place and type of professional activity exercised today (teaching or clinical). From 65 students (54 females and 11 males), 44 are linked in teaching. The most of them work in Sao Paulo and update their CL fre quently. Clinical activities were not often reported in CL.

Descriptors: Pediatric Dentistry. Professional Area Actuation. Dental Education. Graduate.

Correspondência para:

José Carlos Pettorossi Imparato

e-mail: jimparato@usp.br

Faculdade de Odontologia da USP

Av. Prof. Lineu Prestes, 2227

05508-900 - São Paulo/SP 